\title{
Properties of Cryptosystem PGM
}

\author{
Spyros S. Magliveras ${ }^{1}$ \\ Nasir D. Memon
}

University of Nebraska-Lincoln

\begin{abstract}
A cryptographic system, called PGM, was invented in the late 1970's by S. Magliveras. PGM is based on the prolific existence of certain kinds of factorization sets, called logarithmic signatures, for finite permutation groups. Logarithmic signatures were initially motivated by C. Sims' bases and strong generators. Statistical properties of random number generators based on PGM have been investigated in [7], [8] and show PGM to be statistically robust. In this paper we present recent results on the algebraic properties of PGM. PGM is an endomorphic cryptosystem in which the message space is $\mathcal{E}_{|G|}$, for a given finite permutation group $G$. We show that the set of PGM transformations $T_{G}$ is not closed under functional composition and hence not a group. This set is 2-transitive on $\mathcal{Z}_{|G|}$ if the underlying group $G$ is not hamiltonian. Moreover, if $|G| \neq 2^{\circ}$, then the set of transformations contains an odd permutation. An important consequence of the above results is that the group generated by the set of transformations is nearly always the full symmetric group.
\end{abstract}

\section{Introduction}

A Cryptosystem $\Pi$ is an ordered four-tuple $(\mathcal{M}, \mathcal{K}, \mathcal{C}, \mathcal{T})$, where $\mathcal{M}, \mathcal{K}$, and $\mathcal{C}$ are finite sets called the message space, the key space, and the cipher space respectively, and $T$ is a family of transformations $\left\{E_{k}: \mathcal{M} \rightarrow \mathcal{C}, k \in \mathcal{K}\right\}$ such that for each $k \in$ $\mathcal{K}, E_{k}$ is invertible. We denote the inverse of $E_{k}$ by $D_{k}$. Implicit in a cryptosystem $(\mathcal{M}, \mathcal{K}, \mathcal{C}, T)$ is the mapping $E: k \rightarrow E_{k}$ which associates to each key $k \in \mathcal{K}$ the

\footnotetext{
${ }^{1}$ Partially Supported by a US West Communications Grant
} 
transformation $E_{k}$ induced by $k$. The cryptosystem is said to be faithful if $E$ is an injection.

If the message space and cipher space are the same, then the cryptosystem is called endomorphic and in this case for every key $k, E_{k}: \mathcal{M} \rightarrow \mathcal{C}$ is a permutation on $\mathcal{M}$. An endomorphic cryptosystem $I$ is said to be closed if and only if $\mathcal{T}$ is closed under functional composition. In other words, II is closed if and only if for every two keys $i, j \in \mathcal{K}$ there exists a key $k \in \mathcal{K}$ such that $E_{i} E_{j}=E_{k}$. We wish to remark that the term closed had been used by Shannon [9] to mean something totally different. Our usage follows current terminology [4]. Since $\mathcal{T}$ is finite it follows that $\Pi$ is closed if and only if $\mathcal{T}$ forms a group under composition. Let $\mathcal{G}_{\Pi}=\langle\mathcal{T}\rangle$ be the group generated by $\mathcal{T}$ and $\mathcal{S}_{\mathcal{M}}$ the symmetric group on $\mathcal{M}$. Then, of course, $\mathcal{G}_{\mathrm{I}}$ is a subgroup of $\mathcal{S}_{\mathcal{M}}$.

Although the terminology that follows is not standard, it is natural and extends the terminology used in [4]. A Cryptosystem $I$ is said to be $t$-transitive if given any ordered $t$-tuple of distinct messages $\left(m_{1}, \ldots, m_{t}\right) \in \mathcal{M}^{t}$ and any ordered $t$-tuple of distinct ciphertexts $\left(c_{1}, \ldots, c_{t}\right) \in \mathcal{C}^{t}$, there is some $k \in \mathcal{K}$ such that $E_{k}\left(m_{i}\right)=$ $c_{i}(1 \leq i \leq t)$. Here we also write $E_{k}\left(m_{1}, \ldots, m_{t}\right)=\left(c_{1}, \ldots, c_{t}\right)$. Note that we speak of a $t$-transitive system whether or not $T$ is a group. It is clear that a $t$-transitive system is $(t-1)$-transitive.

\section{Logarithmic Signatures and PGM}

If $G$ is a finite permutation group of degree $n$ we call an ordered collection $\alpha=$ $\left\{B_{i}: i=1, \ldots, s\right\}$ of ordered sets $B_{i}=\{u(i, j): j=1, \ldots, r(i)\}$, where $\left|B_{i}\right|$ and $s$ are bounded by a polynomial in $n$, a logarithmic signature of $G$ if each element $g$ of $G$ can be expressed uniquely as a product of the form

$$
g=q_{\bullet} \cdot q_{s-1} \ldots q_{2} \cdot q_{1}
$$

with $q_{i} \in B_{i}$. The elements $q_{i}$ are not necessarily elements of $G$, but could belong to a much larger group in which $G$ is embedded. The $B_{i}$ are called the blocks of $\alpha$ and the vector of block lengths $\mathbf{r}=(r(1), \ldots, r(s))$ is called the type of $\alpha$. We also write $\mathbf{r}=(\alpha)$ for the type of $\alpha$. The logarithmic signature is called non-trivial if $s \geq 2$ and $r(i) \geq 2$ for at least two indices $i$, where $(1 \leq i \leq s)$. Otherwise it is called trivial. A logarithmic signature is called tame if the factorization (1) can be achieved in time polynomial in the degree $n$ of $G$, it is called supertame if (1) can be achieved in time $O\left(n^{2}\right)$. A logarithmic signature is called wild if it not tame. We denote by $\Lambda$ the collection of all logarithmic signatures of $G$.

Let $\gamma: G=G_{0}>G_{1}>\cdots>G_{0}=1$ be a chain of subgroups of $G$, and $\left\{B_{i}: i=1, \ldots, s\right\}$, an ordered collection of subsets of $G$, where each $B_{i}=\{u(i, j):$ 
$j=1, \ldots, r(i)\}$ is a complete set of right coset representatives of $G_{i}$ in $G_{i-1}$. It is easily seen that $\left\{B_{i}\right\}$ forms a logarithmic signature for $G$. Such a logarithmic signature is called transversal with respect to $\gamma$. Here the type $\mathbf{r}=(r(1), \ldots, r(s))$ has $r(i)=\left[G_{i}: G_{i-1}\right]$. In view of the fact that membership in a permutation group can be tested in time polynomial in the degree [3], it is easily seen that a transversal logarithmic signature is tame. We denote the set of all transversal logarithmic signatures of $G$ with respect to a chain $\gamma$ by $\Lambda(\gamma)$. In section 4 we indicate how $\Lambda(\gamma)$ is a single regular orbit under the action of a certain monomial group. Existence of supertame logarithmic signatures is established in the following lemma :

Lemma 2.1 If $G$ is a permutation group then there exists a supertame logarithmic signature for $G$.

Proof: Suppose $G$ acts on the letters of $\Omega=\{1,2, \ldots, n\}$. Let $G=G_{0}>G_{1}>$ $\cdots>G_{a}=1$ be a chain of nested stabilizers in $G$. Thus, $G_{0}=G$ and for $i \geq 1$, $G_{i}$ fixes pointwise the letters $1,2, \ldots, i$ of $\Omega$. Suppose now that the orbit of $i \in \Omega$ under $G_{i-1}$ is $B=\left\{\delta_{1}=i, \delta_{2}, \ldots, \delta_{r(i)}\right\}$ and that $u(i, j) \in G_{i-1}$ moves $\delta_{1}$ to $\delta_{j}$, then $G_{i-1}=G_{i} u(i, 1)+G_{i} u(i, 2)+\cdots+G_{i} u(i, r(i))$. Consider the logarithmic signature

$$
\alpha=[u(1,1), \ldots, u(1, r(1)) ; u(2,1), \ldots, u(2, r(2)) ; \cdots ; u(s, 1), \ldots, u(s, r(s))]
$$

Now, note that an element $h$ in $G_{i-1}$ belongs to the coset $G_{i} u(i, j)$ if and only if $h$ moves $\delta_{1}=i$ to $\delta_{j}$. Thus determining the right $G_{i}$ coset in $G_{i-1}$ to which $h$ belongs requires $O(1)$ operations. The element $h \cdot u(i, j)^{-1}$ fixes $1,2, \ldots, i-1, i$, and therefore belongs to $G_{i}$. Computing $h \cdot u(i, j)^{-1}$ requires $O(n)$ operations. Recursively, given any element $g \in G$, we descend in at most $n$ steps and have $g \cdot u\left(1, j_{1}\right)^{-1} \cdot u\left(2, j_{2}\right)^{-1} \cdots u\left(s, j_{0}\right)^{-1}=1$. Inverting yields the unique factorization $g=u\left(s, j_{1}\right) \cdots u\left(2, j_{2}\right) \cdot u\left(1, j_{1}\right)$.

For the remaining of this paper it is more convenient to write $\{u(i, j): 0 \leq j<$ $r(i) ; 1 \leq i \leq s\}$ rather than $\{u(i, j): 1 \leq j \leq r(i) ; 1 \leq i \leq s\}$ for a logarithmic signature of a group. Before we describe PGM we introduce some notation. By $\alpha[i ; j]$ we mean the $j$ 'th element of the $i^{\prime}$ th block of $\alpha$. Also, if $\mathbf{r}=(\alpha)=(r(1), \ldots, r(s))$ and $\left(p_{1}, \ldots, p_{s}\right) \in \mathcal{Z}_{r(1)} \times \cdots \times \mathcal{Z}_{r(s)}$ then $\alpha\left(p_{1}, \ldots, p_{s}\right):=\alpha\left[s ; p_{s}\right] \cdots \alpha\left[2 ; p_{2}\right] \cdot \alpha\left[1 ; p_{1}\right]$.

If $\mathbf{r}=(r(1), \ldots, r(s))$ is the type of a logarithmic signature $\alpha$, define the integers $m_{i}, i=1,2, \ldots, s$ by :

$$
m_{1}=1, m_{i}=\prod_{j=1}^{i-1} r(j), \quad i=2, \ldots, s .
$$

Let $\lambda$ be the bijection from $\mathcal{Z}_{r(1)} \times \cdots \times \mathcal{Z}_{r(\bullet)}$ onto $\mathcal{Z}_{|G|}$, defined by

$$
\lambda\left(p_{1}, \cdots, p_{\mathbf{s}}\right)=\sum_{i=1}^{\infty} p_{i} m_{i}
$$


then for $x \in \mathcal{Z}_{|G|}, \lambda^{-1}(x)$ is efficiently computable by successive subtractions [representation of $x$ with respect to mixed base $(r(1), \ldots, r(s))]$. For a group $G$ and a logarithmic signature $\alpha=\{\alpha[i ; j]: j=0, \ldots, r(i)-1 ; i=1, \ldots, s\}$ define the bijection $\Theta_{\alpha}: \mathcal{Z}_{r(1)} \times \cdots \times \mathcal{Z}_{r(s)} \rightarrow G$ by

$$
\Theta_{\alpha}\left(p_{1}, \cdots, p_{\bullet}\right)=\alpha\left(p_{1}, p_{2}, \cdots, p_{\iota}\right)
$$

Next, define a map $\hat{\alpha}: \mathcal{Z}_{|G|} \rightarrow G$ by $\hat{\alpha}=\lambda^{-1} \Theta_{\alpha}$. The function $\hat{\alpha}$ is always efficiently computable, but $\hat{\alpha}^{-1}$ is not unless $\alpha$ is tame.

Having defined the mappings $\hat{\alpha}$, for $\alpha \in \Lambda$, the basic cryptographic system PGM is defined as follows : For a given pair of logarithmic signatures, $\alpha, \beta$ with $\beta$ tame, the encryption transformation $E_{\alpha, \beta}$ is the mapping

$$
E_{\alpha, \beta}=\hat{\alpha} \cdot \hat{\beta}^{-1}: \mathcal{Z}_{|G|} \rightarrow \mathcal{Z}_{|G|}
$$

The corresponding decryption transformation is obtained by reversing the order of the pair of logarithmic signatures, that is

$$
D_{\alpha, \beta}=E_{\alpha, \beta}^{-1}=E_{\beta, \alpha}=\hat{\beta} \cdot \hat{\alpha}^{-1}
$$

\section{An Example}

To clarify the ideas presented in the previous section, we illustrate PGM by means of an example. The group used here is the alternating group on five points, $\mathcal{A}_{5}$, of order 60. This implies that the message space $\mathcal{M}$ and the ciphertext space $\mathcal{C}$ are the set $\{0,1, \ldots, 59\}$. A supertame logarithmic signature $\alpha$, with respect to a chain of stabilizer subgroups is obtained by Knuth's algorithm [5] using the generators (1 $\left.\begin{array}{lll}2 & 4 & 5\end{array}\right)$ and $\left(\begin{array}{lll}1 & 2 & 3\end{array}\right)(4)(5)$. Another supertame logarithmic signature $\beta$ is obtained by applying the procedure shuffle to $\alpha$. The blocks of $\alpha$ consist of right coset representatives in a chain of subgroups in $G$. Procedure shuffle consists of changing the coset representatives and their relative order within each block. The number of blocks is $s=3$, and the vector of block lengths is $r=(5,4,3)$. The integers $m_{i}$ are computed to be 1,5 , and 20 respectively. The two logarithmic signatures along with the appropriate knapsack $v$ needed to compute $\lambda$ and $\lambda^{-1}$ efficiently, are shown in Figure (3).

Let us now demonstrate the operation of encipherng. If, for example, the message is 49 , then it can be decomposed uniquely with respect to $v$ as, $49=(4+5+40)$. This process determines the vector of row-indices $\lambda^{-1}(49)=(4,1,2)$. We next compute $\pi=\Theta_{\alpha}(4,1,2)=\alpha[12 ;] \cdot \alpha[7 ;] \cdot \alpha[5 ;]=\left(\begin{array}{lll}1 & 5 & 4\end{array}\right)(2)(3)$. We then compute $\Theta_{\beta}^{-1}(\pi)$, that is, the representation of $\pi$ with respect to $\beta$. Since $\beta$ is supertame and $\pi(1)=5$, we locate the element in block 1 of $\beta$ that sends 1 to 5 . This element is $\beta[5 ;]$. So, 


\begin{tabular}{|c|c|c|}
\hline$\alpha$ & $\mathbf{v}$ & $\bar{\beta}$ \\
\hline$(1)(2)(3)(4)(5)$ & 0 & $(14235)$ \\
\hline$(12345)$ & 1 & $(1)(2)\left(\begin{array}{lll}3 & 5 & 4\end{array}\right)$ \\
\hline$\left(\begin{array}{llll}135 & 524\end{array}\right)$ & 2 & $\left(\begin{array}{lllll}1 & 2 & 5 & 4 & 3\end{array}\right)$ \\
\hline$\left(\begin{array}{lllll}1 & 4 & 2 & 5 & 3\end{array}\right)$ & 3 & $\left(\begin{array}{ll}1 & 3\end{array}\right)(24)(5)$ \\
\hline 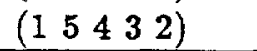 & 4 & $\left(\begin{array}{lllll} & 5 & 3 & 4 & 2\end{array}\right)$ \\
\hline$(1)(2)(3)(4)(5)$ & 0 & $(1)(23)(45)$ \\
\hline$(1)\left(\begin{array}{ll}2 & 3\end{array}\right)(45)$ & 5 & $(1)\left(\begin{array}{lll}2 & 5 & 3\end{array}\right)(4)$ \\
\hline$(1)(243)(5)$ & 10 & $(1)(243)(5)$ \\
\hline$(1)(255)(4)$ & 15 & $(1)(2)(3)(4)(5)$ \\
\hline$(1)(2)(3)(4)(5)$ & 0 & $(1)(2)(3)(4)(5)$ \\
\hline$(1)(2)\left(\begin{array}{lll}3 & 4 & 5\end{array}\right)$ & 20 & $(1)(2)(354)$ \\
\hline$(1)(2)(3 \quad 54)$ & 40 & (1) $(2)(345)$ \\
\hline
\end{tabular}

Figure 1: The two logarithmic signatures and the knapsack

$\pi=h_{1} \cdot \beta[5 ;]$ for some $h_{1} \in G_{1}$. Solving for $h_{1}$, yields $h_{1}=\pi \cdot \beta[5 ;]^{-1}=(1)(2$ 4)(3 5). Now $h_{1}$ fixes 1 and sends 2 to 4 . Now, we locate the element of the second block of $\beta$ which sends 2 to 4 , namely $\beta[8 ;]$. Hence, $h_{1}=h_{2} \cdot \beta[8 ;]$ which yields $h_{2}=(1)(2)(354)$. Continuing in this manner we completely factor $\pi$ with respect to $\beta$ and get $\pi=\beta[11 ;] \cdot \beta[8 ;] \cdot \beta[5 ;]$. This determines the vector of row pointers for $\beta$ to be $(4,2,1) \in \mathcal{Z}_{5} \times \mathcal{Z}_{4} \times \mathcal{Z}_{3}$ and $\lambda(4,2,1)$ is $4+10+20=34$. Thus, we have $E_{\alpha, \beta}(49)=34$. The reader can easily verify that $D_{\alpha, \beta}(34)=E_{\beta, \alpha}(34)=49$.

\section{Transformations on logarithmic signatures}

Suppose that $\beta=\left\{B_{i}: i=1, \ldots, s\right\}$ is a transversal logarithmic signature of a group $G$ with respect to the chain of subgroups $\gamma: G=G_{0}>G_{1} \cdots>G_{1}=1$. Note that while $\beta=\left\{B_{i}: i=1, \ldots, s\right\}$ is a logarithmic signature for $G=G_{0}$, the set of blocks $\beta(k)=\left\{B_{k+1}, \ldots, B_{\text {a }}\right\}$ is a logarithmic signature for $G_{k}$. If the element $u(i, j) \in B_{i}$ of $\beta(k)$ is replaced by $h \cdot u(i, j)$, where $h \in G_{i}$, the resulting collection $\beta(k)^{*}$ forms a new logarithmic signature for $G_{k}$. Moreover, any rearrangement of the elements of a block $B_{i} \in \beta(k)$ yields a new logarithmic signature for $G_{k}$. We call this procedure shuffle.

Procedure shuffle for generating new logarithmic signatures from a given one can be concisely described by considering a certain group action. If $\beta=\left\{B_{i}: i=\right.$ $1, \ldots, s\}, \quad B_{i}=\{u(i, j): j=0, \ldots, r(i)-1\}$ is a transversal logarithmic signature of $G$ with respect to the chain $\gamma: G=G_{0}>G_{1}>\cdots>G_{0}=1$ of subgroups, let $\mathrm{M}$ be the group of all matrices of the form : 


$$
M=\left[\begin{array}{cccc}
H_{1} & 0 & \cdots & 0 \\
0 & H_{2} & \cdots & 0 \\
\vdots & \vdots & & \vdots \\
0 & 0 & \cdots & H_{.}
\end{array}\right]
$$

where $B_{i}$ is an $r(i) \times r(i)$ monomial matrix with entries in $G_{i}$. This means that $B_{i}$ can be thought of as an $r(i) \times r(i)$ permutation matrix whose unity entries have been replaced by arbitrary elements of $G_{i}$. The procedure described above for obtaining new logarithmic signatures of $G$ corresponds to acting on

$$
(u(1,0), \ldots, u(1, r(1)-1) ; u(2,0), \ldots, u(2, r(2)-1) ; \ldots ; u(s, 0), \ldots, u(s, r(s)-1))
$$

on the left by some $M \in \mathbf{M}$, that is,

$$
(v(1,0) \ldots, v(s, r(s)))^{T}=M \cdot(u(1,0), \ldots, u(s, r(s)))^{T}
$$

Thus, the totality $\Lambda(\gamma)$ of logarithmic signatures with respect to $\gamma$ is an $\mathbf{M}$-orbit. We observe that since only the identity of $M$ fixes a logarithmic signature in $\Lambda(\gamma)$, $M$ acts regularly on $\Lambda(\gamma)$. This implies that

$$
|\Lambda(\gamma)|=|\mathrm{M}|=\prod_{i=1}^{\infty}\left|G_{i}\right|^{r(i)} r(i) !=\prod_{i=1}^{\infty}\left(\prod_{j=i+1}^{\infty} r(j)\right)^{r(i)} r(i) !
$$

Now we define another group action on $\Lambda$. Let

$$
\mathbf{T}=G \times \mathcal{S}_{n} \times \mathcal{S}_{n} \times \cdots \times \mathcal{S}_{n} \times G
$$

be a direct product, where $n$ is the degree of $G$ and the the symmetric group $\mathcal{S}_{n}$ occurs $s-1$ times. For $q=\left(g_{0}, g_{1}, \ldots, g_{\mathbf{s}}\right) \in \mathbf{T}$ and

$$
\alpha=(\alpha[1 ; 0], \ldots, \alpha[1 ; r(1)-1] ; \ldots ; \alpha[s ; 0], \ldots, \alpha[s ; r(s)-1]) \in \Lambda
$$

let $q$ act on $\alpha$ by $(q, \alpha) \rightarrow \alpha^{q}$ where

$$
\alpha^{q}=\left(g_{1}^{-1}, \ldots, g_{1}^{-1} ; \ldots ; g^{-1}, \ldots, g_{*}^{-1}\right) \cdot \alpha \cdot\left(g_{0}, \ldots, g_{0} ; \ldots ; g_{,-1}, \ldots, g_{,-1}\right)
$$

This means that all the elements of the first block are multiplied by $g_{1}^{-1}$ on the left and by $g_{0}$ on the right, the elements of the second block are multiplied by $g_{2}^{-1}$ on the left and $g_{1}$ on the right and so on. Finally the elements in block $s$ are multiplied by $g_{0}^{-1}$ on the left and $g_{t-1}$ on the right. It is easily seen that $\alpha^{1}=\alpha$ and $\left(\alpha^{q}\right)^{t}=\alpha^{q t}$, hence $\mathbf{T}$ acts on the collection of logarithmic signatures in $\Lambda$ having $s$ blocks. If $g_{0}=g_{s}=1$, we say that $\alpha^{q}$ is a sandwich of $\alpha$. If $g_{1}=g_{2}=\cdots=g_{s}=1$ then we effectively multiply only the elements of the first block on the right by $g_{0}$. We call this transformation a right translation of $\alpha$. On the other hand if 
$g_{0}=g_{1}=\cdots=g_{0-1}=1$ then we call the transformed logarithmic signature a left translate of $\alpha$. Let $\mathbf{S}$ be the subgroup of $\mathbf{T}$ which consists of the elements of the form $\left(1, g_{1}, \ldots, g_{0-1}, 1\right)$ of $\mathbf{T}$, and $\mathbf{H}$ the subgroup of $\mathbf{S}$ which consists of all elements $\left(1, g_{1}, \ldots, g_{\text {s-1 }}, 1\right)$ with $g_{i} \in G_{i}$.

Suppose that $\alpha$ is a logarithmic signature for a group $G$ of type $(\alpha)=(r(1), \ldots$, $r(i), r(i+1), \ldots, r(s))$. We can create a new logarithmic signature $\beta$ by fusing two consequtive blocks of $\alpha$, say $B_{i}$ and $B_{i+1}$ of lengths $r(i)$ and $r(i+1)$ to a single block of length $r(i) \cdot r(i+1)$. Thus, if $g=q_{s} \cdots q_{i+1} \cdot q_{i} \cdots q_{2} \cdot q_{1}$ is the factorization of $g$ with respect to $\alpha$, then the factorization of $g$ with respect to $\beta$ will be $g=q_{1} \cdots q_{i+2} \cdot t \cdot q_{i-1} \cdots q_{2} \cdot q_{1}$ where $t=q_{i+1} \cdot q_{i}$. In this case we say that $\alpha$ is a refinement of $\beta$. The refinement relation defines a partial order on $\Lambda$ and we write $\alpha<\beta$ to denote that $\alpha$ is a refinement of $\beta$.

Finally, observe that if $g=q_{0} \cdot q_{0-1} \cdots q_{2} \cdot q_{1}$ then we have $g^{-1}=q_{1}^{-1} \cdot q_{2}^{-1} \cdots q_{0}^{-1}$. This implies that if

$$
\alpha=\{\alpha[i ; j]: j=0, \ldots, r(i)-1 ; i=1, \ldots, s\}
$$

is a logarithmic signature then

$$
\alpha^{\prime}=\left\{\alpha[i ; j]^{-1}: j=r(i)-1, \ldots, 0 ; i=s, \ldots, 1\right\}
$$

is also a logarithmic signature. We call $\alpha$ the inversion of $\alpha$. Inversion induces a duality on $\Lambda_{G}$. For example, a system of right coset representatives in a chain $\gamma$ of subgroups (right transversal logarithmic signature), is transformed into a system of left coset representatives (left transversal logarithmic signature). In this paper we do not study properties of inversion.

Definition 4.1 Two logarithmic signatures $\alpha, \beta$ are said to be equivalent if $\hat{\alpha}=\hat{\beta}$.

We denote the equivalence of two logarithmic signatures $\alpha$ and $\beta$ by $\alpha \sim \beta$. If $\alpha, \beta$ and $\gamma$ are logarithmic signatures of a group $G$ then it follows from the definition that $E_{\alpha, \gamma}=E_{\beta, \gamma}$ if and only if $\alpha \sim \beta$. Also, we see that if $\alpha$ is a refinement of $\beta$ then $\alpha \sim \beta$. Hence if two logarithmic signatures are equivalent then they need not have the same type. The concept of equivalence is very important from a cryptanalytic point of view. For a cryptanalyst to break PGM, he only needs to construct logarithmic signatures which are equivalent to the ones specified by the key. The following result was shown by Magliveras and Kreher but its proof has not appeared in print.

Theorem 4.1 Let $\alpha$ and $\beta$ be two logarithmic signatures of a group $G$, which have the same type $\mathbf{r}=(r(1), \ldots, r(s))$. Then $\alpha$ and $\beta$ are equivalent if and only if they are in the same sandwich $\mathbf{S}$-orbit. 
Proof: It is easy to see that if $\alpha$ and $\beta$ are in the same sandwich $\mathbf{S}$-orbit then they are equivalent. For the converse, let $\alpha=\left\{B_{1}, B_{2}, \ldots, B_{0}\right\}$ and $\beta=$ $\left\{B_{1}^{\prime}, B_{2}^{\prime}, \ldots, B_{\imath}^{\prime}\right\}$, where $B_{i}=\left\{u_{i, 1}, u_{i, 2}, \ldots, u_{i, r(i)}\right\}$ and $B_{i}^{\prime}=\left\{u_{i, 1}^{\prime}, u_{i, 2}^{\prime}, \ldots, u_{i, r(i)}^{\prime}\right\}$. Now since $\alpha \sim \beta$, we have

$$
\alpha\left(j_{1}, \ldots, j_{s}\right)=\beta\left(j_{1}, \ldots, j_{s}\right), \quad 0 \leq j_{i}<r(i) .
$$

Specifically, we have $\alpha(j, 0, \ldots, 0)=\beta(j, 0, \ldots, 0)$ for $0 \leq j<r(s)$. That is, $u_{s, j}$. $u_{s-1,0} \cdots u_{1,0}=u_{s, j}^{\prime} \cdot u_{s-1,0}^{\prime} \cdots u_{1,0}^{\prime}$. Let $t_{s-1}=u_{s-1,0}^{\prime} \cdots u_{1,0}^{\prime} \cdot u_{1,0}^{-1} \cdots u_{s-1,0}^{-1}$, then, $u_{x, j}=u_{a, j}^{\prime} \cdot t_{s-1}$, and consequently $B_{s}=B_{a}^{\prime} \cdot t_{s-1}$. In particular note that

$$
u_{s, 0}^{\prime-1} \cdot u_{s, 0}=t_{0-1}^{-1} \text {. }
$$

Next, from (2) we have $\alpha(0, j, 0, \ldots, 0)=\beta(0, j, 0, \ldots, 0)$ for $0 \leq j_{i}<r(i)$. This means that, $u_{s, 0} \cdot u_{s-1, j} \cdot u_{s-2,0} \cdots u_{1,0}=u_{s, 0}^{\prime} \cdot u_{s-1, j}^{\prime} \cdot u_{s-2,0}^{\prime} \cdots u_{1,0}^{\prime}$ therefore, $u_{s, 0} \cdot u_{s-1, j}=$ $u_{a, 0}^{\prime} \cdot u_{s-1, j}^{\prime} \cdot\left(u_{s-2,0}^{\prime} \cdots u_{1,0}^{\prime} \cdot u_{1,0}^{-1} \cdots u_{s-2,0}^{-1}\right)$. Letting $t_{s-2}=u_{s-2,0}^{\prime} \cdots u_{1,0}^{\prime} \cdot u_{1,0}^{-1} \cdots u_{s-2,0}^{-1}$ and using (3) we get $u_{s-1, j}=t_{s-1}^{-1} \cdot u_{s-1, j}^{\prime} \cdot t_{s-2, j}$, hence $B_{s-1}=t_{s-1}^{-1} \cdot B_{s-1}^{\prime} \cdot t_{s-2}$. Continuing in this manner we get $\alpha=\left(t_{1}^{-1}, \ldots, t_{-1}^{-1}, 1\right) \cdot \beta \cdot\left(1, t_{1}, \ldots, t_{0-1}\right)$.

It is easy to see that the monomial shuffle and inversion of a transversal logarithmic signature remain transversal. It is also clear that a subgroup involved in a transversal logarithmic signature can be refined in a 'non-transversal' way. Therefore refinement does not preserve the transversal property. In the cases of sandwiching and left and right translations, although the resulting logarithmic signature is not a transversal, we give a polynomial algorithm for constructing a transversal equivalent to the transformed logarithmic signature in Fig (2). In view of this fact we broaden the use of the term transversal to include logarithmic signatures for which there exists a polynomial time algorithm to compute an equivalent transversal. The algorithm starts with the last block $B$, and standandizes it by multiplying on the right by the inverse of the first element $\alpha[s ; 1]^{-1}$. The resulting block $B$, is the subgroup $G$, with the identity as its first element. We then proceed upwards, standardizing the $j^{\prime}$ th block by multiplying on the left by $\alpha[j-1 ; 1]$ and on the right by $\alpha[j ; 1]^{-1}$. We proceed recursively,finally standardizing the first block by multiplyling on the left by $\alpha[2 ; 1]$. It is easily seen that the resulting logarithmic signature $\beta$ is transversal. Since $\beta$ is a sandwich of $\alpha$, we have $\alpha \sim \beta$. Note that we could have used any element of a block for standardization, rather than the first one. An interesting question which arises is whether there exist new types of transformations which would map a tame logarithmic signature to an equivalent wild one. An answer in the affirmative would lead to a public key cryptosystem based on PGM.

The logarithmic signatures we have mentioned so far have all been transversal and hence tame. A conjecture has been made by the first author that wild logarithmic signatures will occur in profusion for arbitrary groups. The second author 
Input : A logarithmic signature $\alpha=\left\{B_{1}, \ldots, B_{\text {. }}\right\}$ which is the sandwich of a transversal.

Output : A transversal $\beta$ which is equivalent to $\alpha$.

Begin

$$
\begin{aligned}
& x=\alpha[s ; 0]^{-1} ; \\
& x i n v=\alpha[s ; 0] ; \\
& \text { For } j=0 \text { to } r(s)-1 \text { do } \\
& \beta[s ; j]=\alpha[s ; j] \cdot x ; \\
& \text { For } i=s-1 \text { to } 2 \text { do } \\
& \text { begin } \\
& \quad y=\alpha[i ; 0]^{-1} ; \\
& \text { For } j=0 \text { to } r(i)-1 \text { do } \\
& \quad \beta[i ; j]=x i n v \cdot \alpha[i ; j] \cdot y ; \\
& \quad x i n v=y^{-1} ;
\end{aligned}
$$

For $j=0$ to $r(i)-1$ do

$\beta[1 ; j]=x i n v \cdot \alpha[1 ; j]$

End.

Figure 2: Algorithm to construct an equivalent transversal

\begin{tabular}{|c|r|r|c|c|l|}
\hline$G$ & $\left|\hat{\Lambda}_{G}\right|$ & $\left|\mathcal{T}_{G}\right|$ & $\mathcal{G}_{G}$ & $\mu=\left|\mathcal{T}_{G}\right| /|G|$ & comments \\
\hline $\mathcal{Z}_{4}$ & 16 & 24 & $\mathcal{S}_{4}$ & 1.00 & all transversal \\
$\mathcal{V}_{4}$ & 24 & 24 & $\mathcal{S}_{4}$ & 1.00 & all transversal \\
$\mathcal{Z}_{6}$ & 132 & 500 & $\mathcal{S}_{8}$ & 0.69 & all transversal \\
$\mathcal{S}_{3}$ & 288 & 702 & $\mathcal{S}_{6}$ & 0.98 & all transversal \\
$\mathcal{Z}_{8}$ & 1152 & 5568 & $\mathcal{S}_{8}$ & 0.14 & not all transv. \\
$\mathcal{Z}_{4} \times \mathcal{Z}_{2}$ & 2304 & 17088 & $\mathcal{S}_{8}$ & 0.42 & all transversal \\
$\mathcal{V}_{8}$ & 4032 & 10432 & $\mathcal{S}_{8}$ & 0.26 & all transversal \\
$\mathcal{Q}_{8}$ & 1344 & 5280 & $\mathcal{S}_{8}$ & 0.13 & all transversal \\
$\mathcal{D}_{8}$ & 3328 & 32640 & $\mathcal{S}_{8}$ & 0.81 & all transversal \\
$\mathcal{Z}_{9}$ & 648 & 1224 & $\mathcal{S}_{9}$ & 0.01 & all transversal \\
$\mathcal{V}_{9}$ & 2160 & 8208 & $\mathcal{S}_{9}$ & 0.02 & all transversal \\
$\mathcal{A}_{4}$ & 304128 & & $\mathcal{S}_{12}$ & & all transversal \\
\hline
\end{tabular}

Figure 3: Logarithmic Signatures of Small Groups 
has recently constructed many non-transversal logarithmic signatures for the cyclic group $\mathcal{Z}_{8}$ and the alternating group $\mathcal{A}_{5}$. In fact the cyclic group $\mathcal{Z}_{8}$ turns out to be the smallest group for which there exist non-transversal logarithmic signatures.

Finally, we note that the algorithm in Fig (2) can be modified to yield an efficient algorithm to determine whether a given logarithmic signature is a transversal. At every stage, after standardizing the current block $B_{i}$, we check the order of the group generated by $\left\langle B_{i}, B_{i+1}, \ldots, B,\right\rangle$. This can be done in polynomial time from [3] and the fact that the number of generators is polynomial in $n$. If the order equals $r(i) \cdot r(i+1) \cdots r(s)$, we continue otherwise the logarithmic signature is not a transversal. In Figure (3) we tabulate some facts about logarithmic signatures of small groups.

\section{Algebraic properties of PGM}

In the case of PGM we see that the message space and the cipher space is $\mathcal{Z}_{|G|}$ where $G$ is the underlying group, hence PGM is endomorphic. The key space is $\Lambda_{G} \times \Lambda_{G}$ the collection of all ordered pairs of logarithmic signatures of $G$. We denote by $\mathcal{T}_{G}$, the set of transformations defined by the key space and by $\mathcal{G}_{G}$ the group generated by $\mathcal{T}_{G}$ under functional composition. Since sandwiching produces equivalent logarithmic signatures we see that PGM is not faithful. We proceed to discuss some important algebraic properties of PGM.

Lemma 5.1 Given a group $G$ which is not cyclic of prime order, let $x \in \mathcal{Z}_{|G|}$ and $g \in G$. Then there exists a non-trivial logarithmic signature $\alpha \in \Lambda$ such that $\hat{\alpha}(x)=g$.

Proof: Let $|G|=m \cdot p$ where $\mathrm{p}$ is a prime and $m \neq 1$, then there exists a subgroup $P \leq G$ with $|P|=p$. Let $\gamma$ be a transversal logarithmic signature for $G$ with respect to the chain $G=G_{0}>G_{1}=P>G_{2}=1$. Clearly, $\gamma$ has two blocks $B_{1}$ and $B_{2}$, and is of type $\mathbf{r}=(m, p)$. Now, if $g \in G$, then $g \in P u$ for some $u \in B_{1}$, hence $g=q u$ for some $q \in P$. On the other hand, given $x \in \mathcal{Z}_{|G|}$, we have $\lambda^{-1}(x)=(i, j)$ with respect to the type $\mathbf{r}$, where $0 \leq i<m$ and $0 \leq j<p$. Let $\alpha$ be a logarithmic signature which arises from $\gamma$ by rearranging its elements so that $u$ appears in the $i^{\prime} t h$ position of the first block and $q$ in the $j^{\prime}$ th position of the second block. Then we have $\hat{\alpha}(x)=g$.

Theorem 5.1 Given a group $G, x, y \in \mathcal{Z}_{|G|}$, there exist $\alpha, \beta \in \Lambda$ such that $E_{\alpha, \beta}(x)=$ $y$. In other words $\tau_{G}$ is 1-transitive. 
Proof : Let $g$ be an arbitrary element of $G$. From lemma 5.1 there exist $\alpha, \beta \in \Lambda$ such that $\hat{\alpha}(x)=g$ and $\hat{\beta}(y)=g$. This implies that $E_{\alpha, \beta}(x)=\hat{\alpha} \hat{\beta}^{-1}(x)=y$.

Theorem 5.2 If a group $G$ has a proper subgroup $B$, which is not normal in $G$, then $T_{G}$ is 2 -transitive.

Proof : Although $T_{G}$ is not a group, without loss of generality, it will suffice to show that given $x, y \in \mathcal{Z}_{|G|}-\{0\}$, there exist $\alpha, \beta \in \Lambda$ such that $E_{\alpha, \beta}(0, x)=(0, y)$. Let $\alpha$ be a logarithmic signature for $G$ with respect to the chain $G=G_{0}>B=$ $G_{1}>1$. Then $\alpha$ has two blocks $B_{1}$ and $B_{2}$, and type $\mathbf{r}=(k, h)$ where $h=|H|$ and $k=[G: H]$. Given $x, y \in \mathcal{Z}_{|G|}-\{0\}$, let $\lambda^{-1}(x)=\left(i_{x}, j_{x}\right)$ and $\lambda^{-1}(y)=\left(i_{y}, j_{y}\right)$ with respect to $\mathbf{r}$, where $0 \leq i_{x}, i_{y}<k$ and $0 \leq j_{x}, j_{y}<h$. Now we shall consider various cases for the values of $i_{x}, i_{y}, j_{x}, j_{y}$ and in each case construct a logarithmic signature $\beta$ with type $\mathbf{r}$ such that $E_{\alpha, \beta}(0, x)=(0, y)$.

Case 1: $i_{x}, i_{y}, j_{x}, j_{y}>0$

Let $\beta$ be the same as $\alpha$ except that we exchange the elements in positions $i_{x}$ and $i_{y}$ of the first block and the elements in positions $j_{x}$ and $j_{y}$ in the second block. So we have $\beta\left[1 ; i_{y}\right]=\alpha\left[1 ; i_{x}\right]$ and $\beta\left[2 ; j_{y}\right]=\alpha\left[2 ; j_{x}\right]$. It follows that $E_{\alpha, \beta}(0, x)=(0, y)$.

Case $2: i_{z}=i_{y}=0$ and $j_{x}, j_{y}>0$

This can be dealt with in a similar manner as Case 1 . Here we need exchange the elements in the second block only.

Case 3: $i_{x}, i_{y}>0$ and $j_{x}=j_{y}=0$

Again this is similar to Cases 1 and 2. This time we only need to exchange the elements in position $i_{x}$ and $i_{y}$ of the first block.

Case $4: i_{x}=0$ and $i_{y}, j_{x}, j_{y}>0$

Since $H$ is not normal there exist $u \in H$ and $t \in G$ such that $t u t^{-1} \notin H$. Rearrange, if necessary, the second block of $\alpha$ so that $\alpha\left[2 ; j_{z}\right]=u$, and place the identity $1 \in G$ in position $\alpha[1 ; 0]$ as the coset representative of $H$ and also in position $\alpha[2 ; 0]$. So we have $\alpha(x)=\alpha\left[2 ; j_{z}\right] \cdot \alpha[1 ; 0]=u \cdot 1=u \in H$. Now since $v=u^{-1} t u t^{-1} \notin H$, $v$ lies in some coset of $H$, the coset representative of which is $\alpha[1 ; i]$ for some $i \neq 0$. Let $\gamma$ be a logarithmic signature which is obtained from $\alpha$ by (i) replacing $\alpha[1 ; i]$ by $u^{-1} t u t^{-1}$; (ii) exchanging the elements in $\alpha[1 ; i]$ and $\alpha\left[1 ; i_{y}\right]$; (iii) exchanging $\alpha\left[2 ; j_{x}\right]$ and $\alpha\left[2 ; j_{y}\right]$. We ihen right-translate $\gamma$ by $t$ and left-translate it by $t^{-1}$ to obtain a new logarithmic signature $\beta$. The resulting $\beta$ has $\beta[1 ; 0]=t, \beta\left[1 ; i_{y}\right]=u^{-1} t u$ and $\beta[2 ; 0]=t^{-1}, \beta\left[2 ; j_{y}\right]=t^{-1} u$. It follows that $\hat{\beta}(0)=1$ and $\hat{\beta}(y)=u$. Hence $E_{\alpha, \beta}(0, x)=(0, y)$. 
Case $5: i_{y}=0$ and $i_{x}, j_{x}, j_{y}>0$

By Case 4, there exist two logarithmic signatures $\alpha$ and $\beta$ such that $E_{\alpha, \beta}(0, y)=$ $(0, x)$. But then we have $E_{\beta, \alpha}(0, x)=(0, y)$.

Case $6: i_{x}, j_{y}=0$ and $i_{y}, j_{x}>0$

Again as in case 4 we assume that $\alpha[1 ; 0]=\alpha[2 ; 0]=1 \in G$ and $\alpha\left[2 ; j_{x}\right]=u$ where $t^{-1} u t \notin H$. Then we have $\hat{\alpha}(x)=\alpha\left[2 ; j_{x}\right] \cdot \alpha[1 ; 0]=u \cdot 1=u$. Since $v=t^{-1} u t \notin H, v$ belongs to some coset of $H$, with coset representative $\alpha[1 ; i]$ for some $i \neq 0$. Let $\gamma$ be the same as $\alpha$ except that we replace $\alpha[1 ; i]$ by $t^{-1} u t$ and then exchange the elements $\alpha[1 ; i]$ and $\alpha\left[1 ; i_{y}\right]$. We now right-translate $\gamma$ by $t^{-1}$ and left-translate by $t$ to get $\beta$. The resulting $\beta$ has $\beta[1 ; 0]=t^{-1}, \beta\left[1 ; i_{y}\right]=t^{-1} u$ and $\beta[2 ; 0]=t$. It follows that $E_{\alpha, \beta}(0, x)=(0, y)$.

Case $7: i_{y}, j_{z}=0$ and $i_{z}, j_{y}>0$

This is symmetric to Case 6 and follows in the same way as case 5 was obtained from case 4 .

Case $8: j_{x}=0$ and $i_{x}, i_{y}, j_{y}>0$

Again we begin with $\alpha[1 ; 0]=\alpha[2 ; 0]=1 \in G$. Let $\hat{\alpha}(x)=\alpha[2 ; 0] \cdot \alpha\left[1 ; i_{x}\right]=v$ where $v \notin H$. We now construct $\beta$ from $\alpha$ by (i) Exchanging the elements $\alpha\left[1 ; i_{x}\right]$ and $\alpha\left[1 ; i_{y}\right]$; (ii) For $\alpha\left[2 ; j_{y}\right]=u \in H$ replacing $\alpha[1 ; 0]$ by $u^{-1}$; this is possible because $u^{-1} \in H$ and $1, u^{-1}$ represent the same right $H$ coset. (iii) And finally exchinging the elements $\alpha[2 ; 0]$ with $\alpha\left[2 ; j_{y}\right]$. We now have $\beta[1 ; 0]=u^{-1}, \beta\left[1 ; i_{y}\right]=a, \beta[2 ; 0]=u$ and $\beta\left[2 ; j_{y}\right]=1$. It follows that $E_{\alpha, \beta}(0, x)=(0, y)$.

Case $9: j_{y}=0$ and $i_{x}, i_{y}, j_{x}>0$

This is symmetric to the previous case.

Theorem 5.3 Given a group $G, \mathcal{T}_{G}$ is not closed in general

Proof: In Figure (3) we tabulate the size of $\mathcal{T}_{G}$ and $\mathcal{G}_{G}$ for several small groups. It is seen that, in general, $\mathcal{T}_{G} \neq \mathcal{G}_{G}$.

Theorem 5.4 If a group $G$ has a proper subgroup $H$ of odd order then $\mathcal{T}_{G}$ has a transformation which is an odd permutation in $\mathcal{S}_{|G|}$.

Proof : Consider a transversal logarithmic signature $\alpha$ with respect to the chain $G=G_{0}>G_{1}=H>G_{2}=1 . \alpha$ has two blocks and type $\mathbf{r}=(k, h)$, where $h=|H|$ 
and $k=[G: H]$. Now let $\beta$ be a second logarithmic signature which is identical to $\alpha$, except that the first two elements in the first block are interchanged. It is clear that $\hat{\alpha} \hat{\beta}^{-1}$ will be an element of order two and have a factorization as a product of $h$ transpositions. More precisely, we have :

$$
\hat{\alpha} \hat{\beta}^{-1}=(0,1)(k, k+1)(2 k, 2 k+1) \cdots((h-1) k,(h-1) k+1)
$$

In view of the fact that every finite 2-transitive group contains a unique minimal normal subgroup which is elementary abelian or simple [1], [10], the recent classification of finite simple groups yields a classification of finite 2-transitive groups. This in turn leads to the following interesting consequence :

Theorem 5.5 If $G$ is a finite non-hamiltonian group with $|G|$ different from $q,\left(1+q^{2}\right),\left(1+q^{3}\right), \frac{\left(q^{n}-1\right)}{(q-1)}, 2^{n-1}\left(2^{n} \pm 1\right), 11,12,15,22,23,24,176,276$, where $q$ is the power of a prime and $n$ is a positive integer, then $\mathcal{T}_{G}$ is 2-transitive and $\mathcal{G}_{G} \cong \mathcal{S}_{|G|}$.

Proof : The finite doubly transitive groups of degree $m$ are known and consist of the class of alternating groups $\mathcal{A}_{m}$, the class of symmetric groups $\mathcal{S}_{m}$, certain infinite classes of groups of degrees $q,\left(1+q^{2}\right),\left(1+q^{3}\right), \frac{\left(q^{n}-1\right)}{(q-1)}, 2^{n-1}\left(2^{n} \pm 1\right)$, where $q$ is a power of a prime and $n$ a positive integer, and a finite set of certain sporadic groups of degrees $11,12,15,22,23,24,176,276$. Since the degree of $\mathcal{G}_{G}$ is $|G|$, it follows from the hypothesis that $\mathcal{G}_{G}$ must be isomorphic to $\mathcal{A}_{|G|}$ or $\mathcal{S}_{|G|}$. However, since $|G| \neq 2^{a}$, by Theorem 5.4 there is an odd permutation in $\mathcal{G}_{G}$. Hence $\mathcal{G}_{G} \simeq \mathcal{S}_{|G|}$.

\section{Closing Remarks}

Many questions still remain unanswered. We have asked earlier whether there exist transformations which convert a tame logarithmic signature to a wild one. Such a transformation would lead to a public key cryptosystem. Besides, the question whether there exist non-transversal logarithmic signatures which are tame needs to be addressed.

Evidence from Figure (3) suggests that the condition that $G$ possess a nonnormal subgroup may not be necessary for the 2-transitivity of $\tau_{G}$. It would be interesting to investigate whether this condition can be removed.

For encryption purposes being able to factor all of $G$ is not a requirement. For example, for a given $\epsilon>0$, it may be possible to find subsets $X_{1}, X_{2}, \ldots, X_{2}, Z \subset G$ with $|Z| /|G|<\epsilon$ such that each element $g \in G-Z$ has a unique factorization 
$g=x_{1} \cdots x_{2} \cdot x_{1}$, where $x_{i} \in X_{i}$. Such near-factorizations have been studied in [2]

for $Z=\{1\}$, but not much is known for non-abelian $G$ and $Z \neq\{1\}$.

Moreover, we wish to pose the following questions. Is it true that $\mathcal{T}_{G}^{k}$ covers $\mathcal{S}_{G}$, for some fixed integer $k$ independently of $G$, if so, is $k=2$. Finally, is it true that $\tau_{G}$ can be used as a source of random permutations of very large degree.

\section{References}

[1] P. Cameron, Finite permutation groups and finite simple groups, Bull. London Math. Soc., 13(1981), 1-22.

[2] D. de Caen, D. A. Gregory, I. G. Hughes and D. L. Kreher, Near-factors of finite groups, Preprint, (1989).

[3] M. Furst, J. E. Hopcroft, and E. Luks, Polynomial-time algorithms for permutation groups, In Proceedings of the 21 'st IEEE Symposium on Foundations of Computation of Computer Science, (1980), 36-41.

[4] B. S. Kaliski. Jr., R. L. Rivest, and A. T. Sherman, Is the data encryption standard a group? Journal of Cryptology, 1(1988), 3-36.

[5] D. E. Knuth, Notes on efficient representation of permutation groups, Correspondence with $M$. Furst, (1981).

[6] S. S. Magliveras, A cryptosystem from logarithmic signatures of finite groups. In Proceedings of the 29 'th Midwest Symposium on Circuits and Systems, NorthHolland, (1986), 972-975.

[7] S. S. Magliveras and N. D. Memon, The Linear complexity analysis of the cryptosystem PGM, Submitted, (1989).

[8] S. S. Magliveras, B. A. Oberg, and A. J. Surkan, A new random number generator from permutation groups, Red. del Sem Matemat. di Milano, 54 (1985), 203-223.

[9] C. E. Shannon, The mathematical theory of communication, Bell Systems Technical Journal, 28(1949), 379-423.

[10] E. Shult, Permutation groups with few fixed points, In P. Plaumann and K. Strambach, editors, Geometry - Von Staudt's point of view, (1981), 275-311. 